\title{
Infants' Visual Attention to Baby DVDs as a Function of Program Pacing
}

\author{
Alice Ann Howard Gola and Sandra L. Calvert \\ Department of Psychology \\ Georgetown University
}

This study examined the effects of program pacing, defined as the rate of scene and character change per minute, on infants' visual attention to video presentations. Seventy-two infants (twenty-four 6-month-olds, twenty-four 9-montholds, twenty-four 12-month-olds) were exposed to one of two sets of high- and low-paced commercial infant DVDs. Each DVD was approximately 5-min long, and the order the DVDs were viewed was counterbalanced for pace. Attention was higher during rapidly than slowly paced DVDs, particularly for the 6- and 9-month-old infants. These results support previous research documenting that attention is initially controlled by exogenous qualities (e.g., rapid pace), but with development and experience becomes more influenced by endogenous factors.

Examining infants' visual attention is an important step in understanding the cognitive processes infants use to make sense of stimuli in their environments, including the infant DVDs that are now a common experience from the beginnings of life (Rideout \& Hamel, 2006). Attention research demonstrates that infants look at patterns that are complex more than those that are simple for both static (Cohen, 1972; Sigman \& Parmelee, 1974) and dynamic stimuli (Courage, Reynolds, \& Richards, 2006). Our purpose was to examine developmental differences in infant attention to program pacing, that is, the rate of scene and character change, in infant-directed DVDs.

Correspondence should be sent to Alice Ann Howard Gola, Ph.D., Postdoctoral Fellow, Children's Digital Media Center, Georgetown University, 37th and O Streets, NW, 309 White Gravenor, Washington, DC 20057. E-mail: amh243@georgetown.edu 
Stimuli that are high in perceptual salience, which embody characteristics, such as movement, contrast, incongruity, surprise, and complexity, are likely to attract and to hold attention early in development (Berlyne, 1958). For example, infants look longer at checkerboards with more checks than those with fewer checks (Cohen, 1972; Sigman \& Parmelee, 1974). Similarly, infants who were shown both a dynamic (moving) and static (nonmoving) version of a face or a Sesame Street scene looked longer at the dynamic version (Courage et al., 2006). Taken together, the research suggests that infants prefer to look at complex, or perceptually salient, stimuli over more simple stimuli. Nevertheless, complexity is a relative term and the optimal level of complexity can vary by child characteristics, such as their age. For instance, 4-month-old infants do not simply look at the most complex checkerboard; they look instead at checkerboards that are at the right level of complexity for them, as measured by the size of the checkerboard and the number of checks (Cohen, 1972).

Attentional control also improves during the first year of life (Ruff \& Rothbart, 1996). For example, Oakes, Kannass, and Shaddy (2002) gave 6.5- and 10-month-old infants two familiar and two novel toys to play with. During these play sessions, an experimenter activated a highly perceptually salient audiovisual distracter stimulus. The time it took for the 6.5-monthold infants to orient to a perceptually salient distracter object did not vary based on the familiarity or novelty of the original object. However, the 10month-old infants took longer to orient to the perceptually salient distracter when the original object was novel. The implication is that compared to 6.5month-olds, 10-month-olds' attention was more influenced by endogenous variables, as demonstrated by their delayed orientation to a distracter object when they were already fixated on a novel object. Consistent with these findings, Wright and Vlietstra (1975) proposed a major developmental shift in the nature of stimuli that affect young children's attention. In doing so, they created a distinction between exploration and search as a means of gathering information. They argued that attention is initially controlled by the external, perceptually salient features of stimuli (exploration), but increasingly becomes an active search for information with developmental and experiential changes.

Using the exploration to search model, Huston and Wright (1983) examined how perceptually salient television stimuli affect young children's attention. They defined perceptual salience as a property of the stimulus, not the perceiver. They theorized that young children's attention to a television screen is initially controlled by perceptually salient production features, such as rapid pace, fast action, and special effects. With development, children's cognitive level and viewing experiences are expected to lead to a shift in which attention becomes more focused on content, regardless of perceptually 
salient features. Consistent with this model, 6- to 18-month-olds attended more to television scenes that contained more perceptually salient audio and visual features (e.g., special effects) than the 19- to 35-month-olds (Valkenburg \& Vroone, 2004). Similarly, kindergarteners and first graders looked marginally more at high than low-paced programs, but third and fourth graders did not demonstrate these attentional variations (Wright et al., 1984).

Currently, no studies have investigated how program pacing affects infants' attention to the screen. Based on the exploration to search model, we predicted that infants would attend more to rapidly than to slowly paced programs. Due to age-related changes in infants' attentional skills, we expected a shift from exogenous to endogenous control of attention at about 10 months of age (Oakes et al., 2002). Because infants who saw a normal video followed by a distorted video (e.g., backward speech) had significantly longer looks across both videos than those who saw a distorted video first (Pempek et al., 2008), we also expected the order in which infants viewed rapidly and slowly paced DVDs to affect their attention. To capture potential developmental differences, we used a within-subject design to examine how program pacing (high versus low) affected 6-, 9-, and 12-month-olds' attention to the screen.

\section{METHOD}

\section{Participants}

Participants were 72 infants: twenty-four 6-month-olds $(M$ age $=199$ days, $S D=9.75)$, twenty-four 9-month-olds $(M$ age $=289.8$ days, $S D=$ $10.17)$, and twenty-four 12-month-olds $(M$ age $=380.6$ days, $S D=7.5)$, equally distributed by sex. Infants were Caucasian $(n=51)$, Hispanic $(n=3)$, African American $(n=4)$, Asian $(n=2)$, or of other or mixed ethnicities $(n=12) .{ }^{1}$ Their parents' mean educational attainment was 18 years $(S D=2.31)$.

\section{Experimental DVD stimuli}

Fifty-nine infant and toddler DVDs were coded for formal production features; overall, the DVDs were rapidly paced, defined as the number of scene

\footnotetext{
${ }^{1}$ Additional infants were excluded from the sample for accidentally restarting or fastforwarding the video $(n=3)$, crying continuously $(n=3)$, technical problems with the videos of the infants' viewing patterns $(n=2)$, or because the parent regularly directed the infant's attention to the screen $(n=5)$.
} 
and character/object changes divided by the DVD length (Goodrich, Pempek, \& Calvert, 2009). Two rapidly paced DVDs (Baby Signing Time! Volume 1 and Baby Road Trip: City) and two slowly paced DVDs (Here Come the Teletubbies and Eebee's Adventures: Figuring Things Out) were selected from the sample. Rapidly paced DVDs were defined as more complex, a component of perceptual salience, because the rate per minute of scene and character changes was higher than that of the slowly paced videos (see Table 1). All videos involved live characters, and three (Teletubbies, Eebee's, and Baby Road Trip) had muppet characters onscreen.

DVDs were edited so that each was approximately $5 \mathrm{~min}$ in length. Each was then recoded for formal features: pace, character/object action, visual

TABLE 1

Rate per Minute and Proportion of Action, Visual, and Auditory Features for Baby Signing Time (BST), Baby Road Trip (BRT), Teletubbies (TT), and Eebee's (EE)

\begin{tabular}{|c|c|c|c|c|c|c|c|c|}
\hline \multirow[b]{2}{*}{ Feature } & \multicolumn{2}{|r|}{$B S T$} & \multicolumn{2}{|r|}{$B R T$} & \multicolumn{2}{|r|}{$T T$} & \multicolumn{2}{|r|}{$E E$} \\
\hline & Rate & Proportion & Rate & Proportion & Rate & Proportion & Rate & Proportion \\
\hline \multicolumn{9}{|l|}{ Pace } \\
\hline Scene change & 12.50 & - & 8.40 & - & .61 & - & .63 & - \\
\hline $\begin{array}{l}\text { Character/ } \\
\text { object change }\end{array}$ & 14.58 & - & 13.00 & - & .41 & - & .21 & - \\
\hline Total rate of pace & 27.08 & - & 21.40 & - & 1.02 & - & .85 & - \\
\hline \multicolumn{9}{|c|}{ Action } \\
\hline Low & - & .91 & - & .73 & - & .75 & - & .98 \\
\hline Moderate & - & .02 & - & .10 & - & .21 & - & .01 \\
\hline High & - & .06 & - & .12 & - & .04 & - & 0 \\
\hline \multicolumn{9}{|l|}{ Visual } \\
\hline Cuts & 10.00 & - & 10.60 & - & 6.73 & _- & 3.81 & - \\
\hline Special effects & 5.00 & - & 2.60 & - & .41 & - & .63 & - \\
\hline Fades/dissolves & .83 & - & 1.80 & - & .42 & - & .41 & - \\
\hline Zooms & - & .08 & - & .05 & - & 0 & - & 0 \\
\hline Wipes & - & .04 & - & .02 & - & 0 & - & 0 \\
\hline Track and pan & - & .05 & - & .06 & - & 0 & - & 0 \\
\hline \multicolumn{9}{|l|}{ Auditory } \\
\hline Vocalizations & 2.29 & - & 1.2 & - & 8.16 & - & 18.60 & - \\
\hline Rhyming & 0 & - & 0 & - & 0 & - & 0 & - \\
\hline Singing & - & .25 & - & 0 & - & 0 & - & 0 \\
\hline Male language & - & 0 & - & .07 & - & .09 & - & .09 \\
\hline Female language & - & .08 & - & 0 & - & 0 & - & .81 \\
\hline Group language & - & 0 & - & .02 & - & 0 & - & 0 \\
\hline Foreground music & - & 0 & - & .91 & - & 0 & - & .14 \\
\hline Background music & - & 0 & - & 0 & - & .31 & - & .81 \\
\hline Narration & - & .12 & - & .01 & - & .03 & - & .03 \\
\hline Sound effects & .63 & - & 2 & - & .41 & - & 0 & - \\
\hline
\end{tabular}


features (e.g., cuts, special effects), and auditory features (e.g., vocalizations, sound effects; Goodrich et al., 2009). Table 1 includes the proportion of each continuous feature (e.g., singing) by video length and the rate per minute of each discrete feature (e.g., sound effects). Intercoder reliability for formal features across the four videos, as measured by Cohen's kappa, was above .7. $T$ test analyses confirmed that the two rapidly paced videos were significantly higher than the two slowly paced videos on: scene change, $t(2)=-5.33, p<.05$; character/object change, $t(2)=-16.9, p<.05$; and the total rate of pace, $t(2)=-8.12, p<.05$.

Based on the study design of Wright et al. (1984), the four videos were randomly paired to create two sets, each containing one high-paced video and one low-paced video. The sets were: (1) Baby Signing Time and Teletubbies and (2) Baby Road Trip and Eebee's Adventures. As expected, Set 1 and Set 2 did not differ from each other on the mean rate of scene change, $t(2)=.29$, $p=.79$, character/object change, $t(2)=.07, p=.95$, or total rate of pace, $t(2)=.17, p=.88$. Sets also did not differ in the use of action, visual features, or auditory features. However, within the low-paced DVDs, Teletubbies had more camera cuts and sound effects per minute than Eebee's did, and Eebee's had more character vocalizations than Teletubbies did.

\section{Procedure}

Within age and sex groups, infants were randomly assigned to view either Set 1 or Set 2, with the order of presentation counterbalanced for pace. The high- and low-paced DVDs were viewed consecutively in the infants' homes on a laptop computer provided by the experimenter. Infants were seated on a parent's lap, or on the floor with a parent behind them. A video camera was placed directly behind the laptop computer to record the infant's attention to the screen. To increase external validity, infants were observed in their homes where normal distractions, such as toy play, occurred while the infants were viewing.

\section{Attention and behavior scoring}

The videotapes of infants watching the DVDs were later digitized. Using The Observer software by Noldus (Noldus Information Technology, Inc., Leesburg, VA), infants' attention to the screen was coded frame-by-frame as either looking at the screen or not looking at the screen. Visual attention to the screen was calculated as their total duration of looking at the screen divided by the total length of the DVD.

Infants' behavior while their eyes were off-screen was coded as: (1) looking at another person; (2) looking at a toy or other objects; (3) locomoting 
through space; (4) squirming; or (5) other (e.g., fixating on their own hands). The locomotion and squirmy codes overrode the other off-screen looking codes. Eyes-off behavior was calculated as the proportion of time spent looking at a toy, for example, divided by the total amount of time their eyes were off-screen. Intraclass correlations, computed for $25 \%$ of the sample, yielded reliability within the acceptable range of .7-1.0 for all onscreen and off-screen behaviors.

\section{RESULTS}

\section{Percent of looking}

A repeated-measures analysis of variance was conducted on the effect of video pace (high, low), age $(6,9,12$ months), sex (male, female), set $(1,2)$, and pace of the first video (high, low) on infants' percent of looking at the screen. Because infants' attention did not vary by sex, this variable was dropped from all analyses.

A main effect of pace revealed that infants attended to significantly more of the high-paced than the low-placed videos, $F(1,60)=34.60, p<.001$, $\eta_{p}{ }^{2}=.37$ ( $M=68.9 \%$ versus $58.9 \%$, respectively). As seen in Figure 1, there was a trend for the predicted pace by age interaction, $F(2,60)=2.74$, $p=.07, \eta_{p}{ }^{2}=.08$. Post hoc $t$ tests revealed that 6 -month-old infants, $t(23)=3.29, p<.01$, and 9-month-old infants, $t(23)=3.62, p<.01$, looked significantly longer at the perceptually salient high-paced videos than at the less salient low-paced videos. By contrast, 12-month-old infants viewed both the high- and low-paced videos for comparable amounts of time, $t(23)=1.23, p=.23$. No significant age differences occurred in attention to high-paced videos. For low-paced videos, 6-month-old infants did

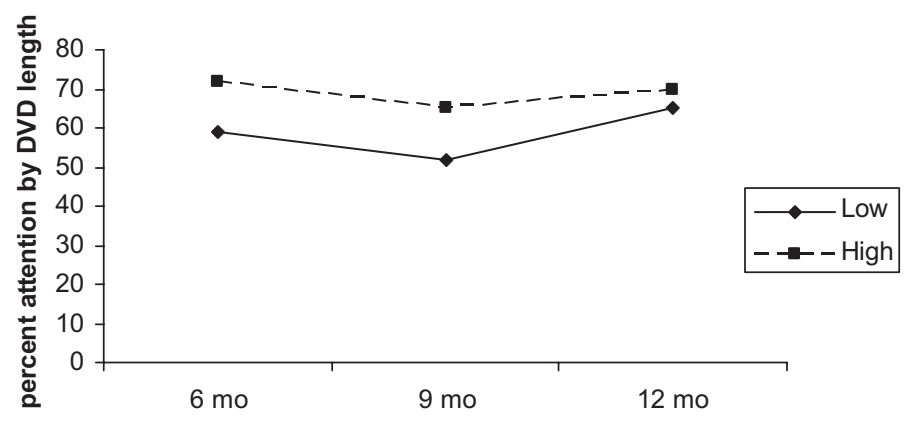

Figure 1 Percent of looking at the screen by DVD pace and age. 
not differ in attention from the 9-month-old infants, $t(46)=1.32, p=.20$, or 12 -month-old infants, $t(46)=-.99, p=.33$. However, the 12-monthold infants viewed significantly more of the low-paced videos than the 9month-old infants, $t(46)=2.55, p<.05$.

A significant interaction between program pace and the pace of the first video viewed also emerged. As seen in Figure 2, infants overall looking time during both videos was significantly affected by the pace of the first video they viewed, $F(1,60)=38.01, p<.001, \eta_{p}{ }^{2}=.39$. Post hoc $t$ tests revealed that if infants viewed a low-paced video first, their visual attention was comparable during both the high- and low-paced videos, $t(36)=-.22, p=.82$. However, if infants viewed a high-paced video first, their attention dropped significantly during the low-paced video, $t(35)=7.40, p<.001$.

A significant effect of set, $F(1,60)=6.34, p<.05, \eta_{p}{ }^{2}=.10$, was qualified by a significant video pace by set interaction, $F(1,60)=4.36, p<.05$, $\eta_{p}{ }^{2}=.07$. The less salient low-paced videos created variations in visual attention, but the high-paced videos did not. Specifically, infants who viewed Eebee's, the low-paced video from Set 2, paid significantly less attention than those who viewed Teletubbies, the low-paced video from Set 1 $(52 \%$ versus $65 \%), t(70)=2.95, p<.01$.

\section{Eyes off the screen behavior}

Approximately $70 \%$ of infants' off-screen time was spent looking at another person or at a toy. A multivariate analysis of variance examined whether infants' behaviors while their eyes were off-screen explained the difference in attention to the low-paced videos between the 9- and 12-month-olds, but not the 6- and 12-month-olds. The proportions of each behavior while infants' eyes were off-screen during the low-paced videos were entered as the

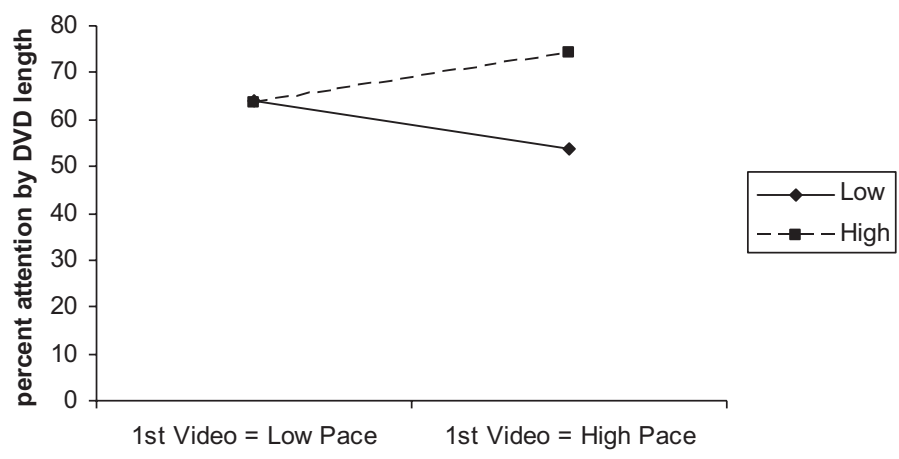

Figure 2 Percent of looking at the screen by DVD pace and order. 
dependent variables (i.e., looking at another person, looking at a toy, locomoting, squirming, or other). The independent variable was age (6-, 9-, or 12-month-old).

A main effect of age emerged on infants' locomotion while their eyes were off-screen during a low-paced video, $F(2,69)=3.91, p<.05, \eta_{p}{ }^{2}=.10$. Post hoc Tukey tests revealed that the 9- and 12-month-olds locomoted significantly more than the 6 -month-olds $(7.1 \%, 7.8 \%$, and $0 \%$, respectively), making the 6-month-olds a more captive audience.

\section{DISCUSSION}

The purpose of this study was to examine the effect of program pacing on infants' visual attention to commercially made DVDs. As predicted, infants watched significantly more of the rapidly paced than the slowly paced videos. Infants' attentional patterns to high-paced over low-paced videos supports previous research documenting infants' preferences for more complex, perceptually salient stimuli over simple nonsalient stimuli (Berlyne, 1958; Courage et al., 2006; McCall \& Kagan, 1967; Sigman \& Parmelee, 1974; Valkenburg \& Vroone, 2004).

The exploration to search model predicts that young children's attention is initially governed by perceptually salient production qualities, but with development and experience, attention becomes increasingly controlled by endogenous factors (Huston \& Wright, 1983; Wright \& Vlietstra, 1975). This developmental prediction was supported at marginal levels of significance. As predicted, the attentional preference for high- over low-paced programs only emerged at ages 6- and 9-months, not for the 12-month-olds. By 12 months of age, infants viewed just as much of the slowly paced DVDs as the rapidly paced DVDs. Consistent with these findings, Oakes et al. (2002) found that attention changes from exogenous to more endogenous control at around age 10 months. Taken together, the research suggests that $10-$ 12 months may be the beginning of the developmental time frame when perceptual salience is no longer the key driver of very young children's visual attention.

The 6-month-old infants in our study, however, viewed as much of the low-paced videos as the 12-month-old infants did. We propose the following possible explanation for this unexpected finding. While the 12month-olds locomoted significantly more than the 6-month-olds, the increased mobility did not lead to a reduction in attention for the 12month-old infants. By contrast, the 9-month-olds locomoted at a comparable rate to the 12 -month-old infants $(7.1 \%$ versus $7.8 \%$, respectively), and the 9-month-old infants watched significantly less of the low-paced 
DVDs than the 12-month-old infants did. The current findings suggest that the 12-month-old infants' mobility did not disrupt their attention to the low-paced DVD, perhaps because their attention was more purposeful than that of the 9-month-old infants (see also Oakes et al., 2002). Alternatively, 6-month-olds may have watched more of the low-paced videos than the 9-month-olds simply because they could not leave the viewing situation.

The order in which the DVDs were viewed also influenced subsequent attentional patterns. Viewing a rapidly paced video first disrupted infants' attention to a subsequent slower paced video. However, if they viewed the low-paced video first, infants paid similar amounts of attention to the lowpaced and high-paced video. It is possible that infants' drop in attention during a subsequent low-paced video is a result of the high-paced videos being more stimulating than the low-paced videos. However, if boredom during the low-paced videos caused the decrease in attention, we would expect infants' attention to increase during a high-paced DVD if viewed after a low-paced DVD. That did not occur. It might be that the high-paced DVDs are harder to process and thus overload very early attentional capabilities, causing a decline in attention during subsequent DVD exposure, at least in the short term.

One limitation is that it is too simplistic to assume that the relation between attention and pacing is inevitably linear for infants and children. We found here that 12-month-old infants watched the same amount of the rapidly and slowly paced programs. When examining programs designed for an older audience, however, kindergarten and first graders looked marginally longer at high-paced than low-paced programs, but third and fourth graders did not (Wright et al., 1984). Thus, the optimal level of program pacing, and complexity, for enhanced attention depends on age, features, content, and the specific programs viewed (Bickham, Wright, \& Huston, 2001). Put another way, the rapidly paced infant-directed DVDs may have presented the optimal level of pacing and complexity for the infants in our study. Anything more complex, or faster paced, such as an adult action film, might have led to a reduction in infant attention. Other perceptually salient features that commonly co-occur with rapid pace could also partly explain infants' enhanced attention for the high- over the low-paced DVDs. For instance, children's Saturday morning television programs were characterized by a cluster of features involving rapid scene changes, fast action, foreground music, sound effects, and character vocalizations (Huston et al., 1981). For the slowly paced videos, infants viewed significantly less of Eebee's than of Teletubbies. Since Teletubbies and Eebees' differed in the other formal features present, it is unclear whether the features or the content contributed to this difference in attention. Finally, infants were studied 
in their homes to increase external validity, but internal validity challenges (e.g., noise, siblings, pets) occurred because the testing environment was not the same.

In conclusion, infants attend to novel perceptually salient events to explore and learn in the first year of life (Ruff \& Rothbart, 1996). Rapidly paced infant DVDs are now a part of those early experiences (Goodrich et al., 2009). Our research demonstrates that rapidly paced, perceptually salient infant videos are garnering the attentional interest of infants. Although infants can learn from simple experimentally made video stimuli (Barr, Wyss, \& Somander, 2009), how much infants learn from rapidlypaced commercially made DVDs remains an unanswered question.

\section{ACKNOWLEDGMENTS}

We thank the infants and parents who participated in this study. We also thank the members of the Children's Digital Media Center team for their assistance with this project, particularly Marta Perez, Alex Verdaguer, Christina Baker, Monica Perrigino, and Mollie Schmitz who helped collect and code data. We gratefully acknowledge the support for this research by Grant \#0623871 from the National Science Foundation.

\section{REFERENCES}

Barr, R., Wyss, N., \& Somander, M. (2009). The influence of electronic sound effects on learning from televised and live models. Journal of Experimental Child Psychology, 103, 1-16.

Berlyne, D. E. (1958). The influence of complexity and novelty in visual figures on orienting responses. Journal of Experimental Psychology, 55, 289-296.

Bickham, D., Wright, J. C., \& Huston, A. C. (2001). Attention, comprehension, and the educational influences of television. In D. G. Singer \& J. L. Singer (Eds.), Handbook of children and the media (pp. 101-134). London: Thousand Oaks.

Cohen, L. B. (1972). Attention-getting and attention-holding processes of infant visual preferences. Child Development, 43, 869-879.

Courage, M. L., Reynolds, G. D., \& Richards, J. E. (2006). Infants' attention to patterned stimuli: Developmental change from 3 to 12 months of age. Child Development, 77, 680-695.

Goodrich, S., Pempek, T., \& Calvert, S. L. (2009). Formal production features of infant and toddler DVDs. Archives of Pediatrics and Adolescent Medicine, 163, 1151-1156.

Huston, A. C., \& Wright, J. C. (1983). Children's processing of television: The informative functions of formal features. In J. Bryant \& D. R. Anderson (Eds.), Children's understanding of television: Research on attention and comprehension (pp. 35-68). New York, NY: Academic Press, Inc.

Huston, A. C., Wright, J. C., Wartella, E., Rice, M. L., Watkins, B. A., Campbell, T., \& Potts, R. (1981). Communicating more than content: Formal features of children's television programs. Journal of Communication, 31, 32-48. 
McCall, R., \& Kagan, J. (1967). Attention in the infant: Effects of complexity, contour, perimeter, and familiarity. Child Development, 38, 939-952.

Oakes, L., Kannass, K., \& Shaddy, D. (2002). Developmental changes in endogenous control of attention: The role of target familiarity on infants' distraction latency. Child Development, 73 , $1644-1655$.

Pempek, T. A., Kirkorian, H. L., Stevens, M., Lund, A. F., Richards, J. E., \& Anderson, D. R. (2008, March). Video comprehensibility and attention in very young children. Poster presented at the biennial International Conference for Infant Studies, Vancouver, Canada.

Rideout, V., \& Hamel, R. (2006). The media family: Electronic media in the lives of infants, toddlers, preschoolers, and their parents. Menlo Park, CA: Henry J. Kaiser Family Foundation.

Ruff, H. A., \& Rothbart, M. K. (1996). Attention in early development. New York, NY: Oxford University Press.

Sigman, M., \& Parmelee, A. (1974). Visual preferences of four-month-old premature and full-term infants. Child Development, 45, 959-965.

Valkenburg, P., \& Vroone, M. (2004). Developmental changes in infants' and toddlers' attention to television entertainment. Communication Research, 31, 288-311.

Wright, J. C., Huston, A. C., Ross, R., Calvert, S. L., Rollandeli, D., Weeks, L. A., ... Potts, R. (1984). Pace and continuity of television programs: Effects on children's attention and comprehension. Developmental Psychology, 20, 653-666.

Wright, J. C., \& Vlietstra, A. (1975). The development of selective attention: From perceptual exploration to logical search. In H. W. Reese (Ed.), Advances in child development and behavior (Vol. 10, pp. 195-239). New York, NY: Academic Press. 